\section{Mauriac Syndrome in the Age of Newer Insulin Analogues}

\section{Abstract}

The authors describe a five year-old plump looking female child with poorly controlled type 1 diabetes mellitus, hepatomegaly and growth retardation. Contrary to the usual phenotype of thin emaciated appearance in type 1 diabetes mellitus, the presence of above features should make one suspect Mauriac syndrome. Since the basis of this syndrome is poor metabolic control, early recognition is important. The authors emphasize the importance of optimal glycaemic control in preventing complications associated with Mauriac syndrome.

Keywords: Mauriac syndrome; Children; Type 1 diabetes

Received: November 05, 2015; Accepted: December 12, 2015; Published: December 22, 2015

\section{Introduction}

A syndrome of uncontrolled type 1 diabetes, growth restriction and hepatomegaly which used to be commonly referred to as the Mauriac syndrome during the pre long acting insulin era is being increasingly reported of late albeit sporadically [1-4]. Although they are growth restricted, they look cushingoid due to excess deposition of fat and glycogen giving them a plump look (Figure 1) in contrast to children with type-1 diabetes who otherwise look thin and wasted. We report a five year old girl who presented with uncontrolled diabetes and ketoacidosis with features of Mauriac syndrome

\section{Brief Report}

A five year old girl diagnosed with type 1 diabetes since one year of age and on mixtard insulin with inadequate dosing and poor compliance due to monetary constraints, was brought with history of fever of one day duration, vomiting and fast breathing. Examination revealed a Glasgow coma scale of 13/15, features of severe dehydration with elevated blood sugar and ketoacidosis. She was managed as diabetic ketoacidosis with intravenous fluids and insulin infusion as per Milwaukee protocol. On examination she looked plump and chubby but her weight and height were less than third centile. Her anthropometric parameters were apparently normal from birth till one year of age, when she was diagnosed with the disease and since then she has not been growing well. She had a firm hepatomegaly with a span of 11 $\mathrm{cm}$. Investigations revealed $\mathrm{HbA} 1 \mathrm{C}$ concentration of $16.1 \%$. Liver function tests were normal except for a mildly elevated AST (AST/ALT 53/20 IU/L, Bil 0.7 mg/dl, STP/Alb 7.8/3.9). Fasting lipid profile showed hypercholesterolemia (Total cholesterol $231 \mathrm{mg} /$

\section{Venkatesh Chandrasekaran, Revanth Baineni, Srinivasaraghavan Rangan, Venkatesh Chandrasekaran and Vishnu Bhat}

\author{
DNB, Pediatrics, MNAMS, JIPMER, \\ Pondicherry, India
}

\section{Corresponding Author:}

Venkatesh Chandrasekaran

झ cvenkatesh@hotmail.com

DNB, Pediatrics, MNAMS, JIPMER, Pondicherry, India.

Citation: Chandrasekaran V, Baineni R, Rangan S, et al. Mauriac Syndrome in the Age of Newer Insulin Analogues. J Pediatr Care. 2015, 1:1.

dl, Triglycerides 139 mg/dl, LDL 143 mg/dl, HDL 66 mg/dl). Early morning serum cortisol was $16.56 \mathrm{mcg} / \mathrm{dL}$ (Lab reference: 6 to 23 micrograms per $\mathrm{dL}$ ). Thyroid function tests were normal (TSH 2.69, FT4 0.93). Ultrasound abdomen showed mild hepatomegaly.

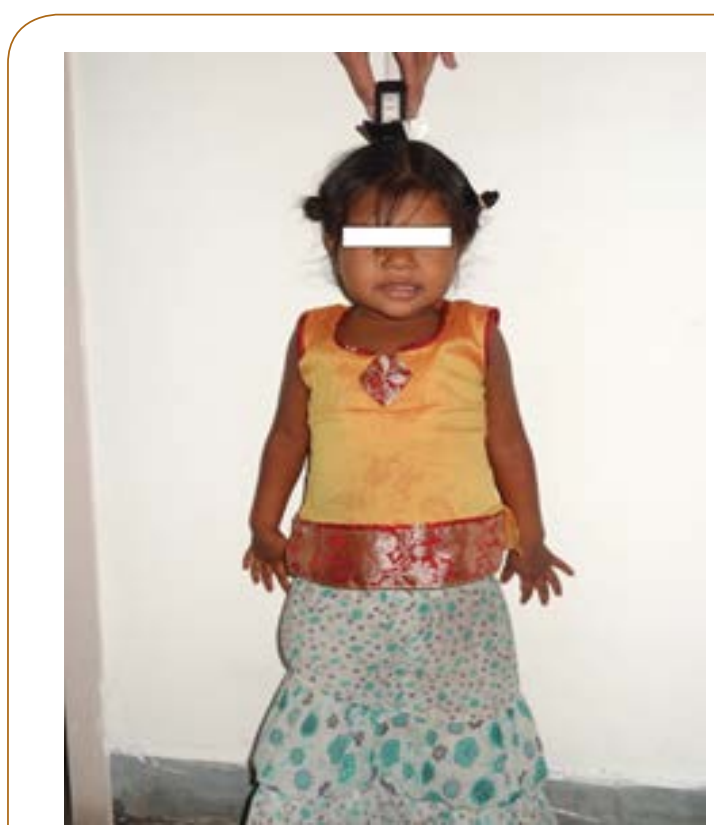

Figure 1 Chubby looking short child with Mauriac syndrome. 
Optic fundus examination was normal. The child was managed with subcutaneous Mixtard insulin regimen at $0.8 \mathrm{U} / \mathrm{Kg} /$ day with diet modification as the parents could not afford glargine injection. The blood glucose was fluctuating for a period of four days and was gradually controlled with $1 \mathrm{U} / \mathrm{Kg} /$ day of insulin. She was discharged and advised to monitor capillary blood glucose regularly at home and to follow up regularly in our endocrine clinic and plan to shift him to glargine at the earliest available opportunity.

\section{Discussion}

Mauriac syndrome is an uncommon complication of uncontrolled type 1 diabetes reported in children belonging to age group of 13-17 years [5]. Hepatomegaly is common with mild elevation of hepatic transaminase. Enlarged liver cells with glycogen deposition, steatosis and fibrosis are the usual pathologic features of this condition. Transaminase levels closely correlate with $\mathrm{HbA} 1 \mathrm{c}$ levels and may be a potential prognostic marker [6]. The growth failure is thought to be due to a combination of factors including low insulin like growth factor-1 levels (IGF-1) and failure of glucose utilization. With institution of proper care and insulin treatment, the growth failure and other metabolic abnormalities can be reversed to the pre illness state $[7,8]$. Nevertheless, it should be remembered that over-zealous correction of insulin deficiency might result in rapid deterioration of retinopathy and nephropathy [7]. Therefore a balanced approach is warranted in managing such patients. Newer insulin analogues and using basal bolus regimen with prandial short acting insulin can result in optimal glycemic control in these children. The decision to use a particular insulin analogue should be individualized depending on pharmaco-economics rather than being protocol centric. If sustaining a newer insulin analogue like glargine is a problem, the readily available insulin premix can still be used as a bargain to ensure good compliance rather than striving for perfect control and risking poor compliance. Enrolling diabetics under state sponsored insurance schemes can be a viable option to ensure sustainable therapy with no cost to the patient. In conclusion, Mauriac syndrome continues to exist despite newer insulin regimens. Ensuring compliance to therapy, providing nutritional support and good follow-up care can help to mitigate most of the complications associated with this syndrome. 


\section{References}

1 Mahesh S, Karp RJ, Castells S, Quintos JB (2007) Mauriac syndrome in a 3 year old boy. Endocr Pract 13: 63-6.

2 Patidar PP, Philip R, Saran S, Gupta KK (2012) A rare case of Mauriac syndrome. Indian J Endocrinol Metab 16: 486-7.

3 Gutch M, Philip R, Saran S, Tyagi R, Gupta KK (2013) Re-emergence of a rare syndrome: A case of Mauriac syndrome. Indian J Endocrinol Metab 17: s283-5.

4 Elder CJ, Natarajan A (2010) Mauriac syndrome-a modern reality. J Pediatr Endocrinol Metab 23: 311-3.
5 Dias J, Martin S, Caravalho S, Marques O, Antunes A (2013) Mauriac syndrome still exists. Endocrinol Nutr 60: 245-8.

6 Fitzpatrick E, Cotoi C, Quaglia A, Sakellariou S, Ford-Adams ME, Hadzic N (2014) Hepatopathy of Mauriac syndrome: a retrospective review from a tertiary liver centre. Arch Dis Child 99: 354-7.

7 Kim MS, Quintos JB (2008) Mauriac syndrome: growth failure and type 1 diabetes mellitus. Pediatr Endocrinol Rev 5: 989-993.

8 Shrivastava MS, Palkar AV, Padwal NJ, Moulick N (2011) Reversible steatohepatosis in a young boy with brittle type 1 diabetes mellitus: Mauriac syndrome. BMJ Case Rep 2011: bcr0320113975. 